\title{
Susceptibility and \\ Resistance of Various Strains of Mycoplasma hyorhinis to Antisera, Polymyxins and Low pH Values
}

\author{
By Z. DINTER \\ Institute of Virology, Royal Veterinary College and \\ National Veterinary Institute, Stockholm, Sweden \\ AND D. TAYLOR-ROBINSON \\ M.R.C. Clinical Research Centre, Harvard Hospital, Salisbury, Wiltshire
}

(Accepted for publication I6 April 1969)

\section{SUMMAR Y}

The growth of some strains of Mycoplasma hyorhinis on solid medium was inhibited by rabbit antisera incorporated in filter-paper discs. Other strains were resistant to the same antisera. Rings of precipitate were observed in the agar around the discs. The largest number of precipitation rings and the most intense ones occurred with antiserum-sensitive strains. The antiserumsensitive strains were also more sensitive than the resistant strains to colistin and polymyxin B when these antibiotics were incorporated in paper discs. Colonies of antiserum-resistant strains developed on solid medium containing low concentrations of horse serum, while colonies of sensitive strains developed only on solid medium containing higher concentrations of serum. The growth of antiserum-sensitive strains, but not resistant ones, was suppressed on solid medium at $\mathrm{pH}$ 6.5. All these differences between sensitive and resistant strains were only expressed on solid medium; the phenomena were not observed when the organisms were grown in liquid medium. It is suggested that the differences between sensitive and resistant strains are due to changes in the composition of the mycoplasma membranes.

\section{INTRODUCTION}

Mycoplasma hyorhinis (Switzer, 1955) may be identified by various serological tests. However, in disc growth-inhibition tests on agar medium (Clyde, I964) some strains were not inhibited by antisera against $M$. hyorhinis (Dinter, Danielsson \& Bakos, 1965). In similar tests, Hayflick \& Stanbridge (1967) also observed resistance to antibodies and ascribed this to antigenic shifts occurring in organisms growing on agar medium. We have examined in greater detail the resistance and susceptibility to anti- $M$. hyorhinis sera of various strains of $M$. hyorhinis and have observed other biological phenomena with the same strains. The results show that these phenomena occur only when the organisms are grown on solid media and not when they are grown in liquid media.

\section{METHODS}

Mycoplasma media. The standard liquid medium consisted of beef-heart infusion with addition of $10 \%(\mathrm{v} / \mathrm{v})$ extract of baker's yeast, I \% (w/v) glucose, $20 \%(\mathrm{v} / \mathrm{v})$ unheated horse serum, penicillin ( $100 \mathrm{u} . / \mathrm{ml}.), 0.05 \%(\mathrm{w} / \mathrm{v})$ thallium acetate and 
$0.002 \%(w / v)$ phenol red, adjusted to $\mathrm{pH} 7 \cdot 8$. The extract of baker's yeast was prepared according to the method of Hayflick (1965). The standard agar medium was composed of the same ingredients except that glucose was decreased to $0.1 \%$ and phenol red omitted; the agar (Noble, Difco) was $1 \%(w / v)$, and the medium was adjusted to $\mathrm{pH} 7.5$. The agar medium, poured in plastic Petri dishes, was used within I to 3 days after being prepared.

Mycoplasma strains. Nine strains of Mycoplasma hyorhinis were studied. Five of them (F, S7, 200, SK, G) were used in a previous study (Dinter et al. I965). Four other strains (F/D, K, GDL, SWITZER 9832, here called ' $W$ ') were added to this group. $F / D$ is an antiserum-insensitive derivative of the $F$ strain. The strains $\mathrm{W}, \mathrm{K}$ and GDL were kindly supplied by Drs D. G. ff. Edward (Wellcome Research Laboratories, Beckenham, Kent, England), K. Bakos (National Veterinary Institute, Stockholm, Sweden) and R. H. Purcell (National Institutes of Health, Bethesda, U.S.A.), respectively. Stock cultures of the strains in liquid medium were kept at $-60^{\circ}$.

Cultivation. All cultures were incubated at $37^{\circ}$, agar cultures being placed in containers with moist air.

Millipore filtration. Liquid cultures were filtered through membranes (Millipore Filter Co., Bedford, Mass., U.S.A.), of average pore diameter $650 \mathrm{~m} \mu, 450 \mathrm{~m} \mu$, $300 \mathrm{~m} \mu$ and $220 \mathrm{~m} \mu$, by using a Swinny adaptor. The number of organisms before and after each filtration was measured.

Antibiotics. The following preparations of polymyxin were used: polymyxin B (Novo Industri, Copenhagen) and colistin sodium methanesulphonate ( $H$. Lundbeck \& Co., Copenhagen-Valby, Denmark). These antibiotics were diluted in liquid medium of $\mathrm{pH} 7.8$ to produce concentrations indicated in Tables 5 and 6. Paper discs $(6 \mathrm{~mm}$. diameter) containing various antibiotics (Department of Bacteriology, Karolinska Sjukhuset, Stockholm) were also used. The following were used, with the amounts/disc indicated in parentheses: bacitracin (IO i.u.), chloromycetin ( $30 \mu \mathrm{g}$.), colistin sodium methanesulphonate (900 i.u.), erythromycin (50 $\mu \mathrm{g}$ ), gentamycin (10 $\mu \mathrm{g}$.), kanamycin (5 $\mu \mathrm{g}$.), neomycin (50 $\mu \mathrm{g}$ ), novobiocin (50 $\mu \mathrm{g}$.), polymyxin B (50 $\mu \mathrm{g}$.), streptomycin $(50 \mu \mathrm{g}$.), terramycin (50 $\mu \mathrm{g}$.), tetracycline (50 $\mu \mathrm{g}$.), vibramycin $(50 \mu \mathrm{g}$.).

Rabbit antisera. Antisera to 8 strains of Mycoplasma hyorhinis were available, some of them having been used in a previous study (Dinter et al. 1965). These antisera, stored at $-20^{\circ}$, were used after being heated for $30 \mathrm{~min}$. at $56^{\circ}$ and absorbed with a Io-fold concentrate of liquid medium as described by Dinter et al. (I965).

Gel diffusion. Antigen production and the microtechnique were as described previously (Dinter et al. 1965).

Metabolic inhibition (m.i.). For tests with antisera this test was done by a microtechnique as described previously (Taylor-Robinson, Purcell, Wong \& Chanock, 1966). The results were recorded when a colour change equivalent to about half a $\mathrm{pH}$ unit had occurred in the wells containing mycoplasma organisms but no antiserum. In tests with colistin, the technique was modified and was done in tubes with $\mathrm{I} \mathrm{ml}$. medium/tube. Each mycoplasma strain was diluted in serial Io-fold steps in the presence or absence of various amounts of colistin in the medium. The results were read when the colour change in the titration series without colistin had ceased to progress. For both types of test one colour-changing unit (c.c.u.) of mycoplasma activity was defined as the highest dilution of organisms which produced a colour change. 
Disc growth-inhibition. The technique was that of Clyde (1964). Stock mycoplasma cultures, previously titrated, were diluted in medium so that $0.1 \mathrm{ml}$. contained about $10^{5}$ colony-forming units (c.f.u.). This was spread on the agar medium which had been dried for $45 \mathrm{~min}$. at $37^{\circ}$ before inoculation. Filter paper discs of $6 \mathrm{~mm}$. diameter were soaked with $0.025 \mathrm{ml}$. of undiluted antiserum and were placed on the agar. The same technique was used with discs containing antibiotics. Zones of inhibition were measured from the edge of the disc to the edge of the area of colonies as soon as these were visible.

\section{RESULTS}

Identification of mycoplasma strains. The various strains of Mycoplasma hyorhinis were identified in preliminary gel-diffusion tests. Lines of precipitate formed between a well which contained a mycoplasma antigen and a well which contained its specific antiserum, and reactions of identity were seen with all strains tested against such an antiserum. All nine strains of $M$. hyorhinis were examined also by the m.i. technique, and there was a sufficiently close relationship between them to permit identification by this technique. The results of testing six strains against each of their respective antisera are shown in Table $\mathrm{I}$. The metabolism of all strains was inhibited by a single antiserum. Each of the antisera had a high antibody titre against its homologous organism, although an antiserum sometimes inhibited a heterologous strain only at a low titre. All other mycoplasma species antisera that were tested failed to inhibit the glucose metabolism of the F strain of $M$. hyorhinis, and an antiserum to this strain did not inhibit these other mycoplasma species (Taylor-Robinson \& Dinter, 1968).

Table I. Relationship between six strains of Mycoplasma hyorhinis as shown by the metabolic-inhibition technique

\begin{tabular}{lrrrrrr} 
& \multicolumn{5}{c}{ Reciprocal of m.i. titre with rabbit antisera to } \\
\cline { 2 - 7 } Strain & $F(I)^{*}$ & $S 7$ & 200 & SK & G & GDL \\
F & 10240 & 80 & 10240 & 640 & 5120 & 2560 \\
S7 & 640 & 640 & 5120 & 10240 & 5120 & 5120 \\
200 & 2560 & 2560 & 20480 & 5120 & 10240 & 5120 \\
SK & 160 & 160 & 1280 & 2560 & 160 & 160 \\
G & 2560 & 640 & 1280 & 1280 & 1280 & 2560 \\
GDL & 320 & 160 & 5120 & 10240 & 1280 & 10240 \\
& $*$ & &
\end{tabular}

Sensitivity of Mycoplasma hyorhinis strains to antisera. Six strains and their respective antisera were tested comparatively by metabolic and disc growth-inhibition. In m.i. tests, the metabolism of each strain was inhibited by each antiserum, that against strain $\mathbf{5} 7$ having the weakest activity (Table I). However, in disc growth-inhibition tests the growth of all strains was not inhibited (Table 2); two strains were inhibited by five antisera, two strains by three antisera and two strains were not inhibited by any of the antisera. Also, antiserum to strain 57 did not inhibit the growth of any of the strains. These results were thought to reflect differences in the strengths of antisera and in the sensitivity of particular strains to antibodies, especially when tested by the disc growth-inhibition method.

To examine more fully the sensitivity of the strains to antisera in the discs, growth- 
inhibition tests were made with all the nine mycoplasma strains and their antisera. The results are summarized in Table 3; these show that antisera to strains 200, SK and GDL were strongest in that they inhibited the growth of more strains than did the other six antisera. Further, the growth of strain $\mathrm{K}$ was inhibited by all antisera, including the weakest antiserum (that to strain $\mathrm{s} 7$ ). The $\mathrm{K}$ strain was thus regarded as being the most 'sensitive'. Within the group of sensitive strains, strain GDL appeared to be least sensitive. The growth of four strains (G, W, s7, F/D) was not inhibited by any antiserum and these were regarded as being 'insensitive' strains.

Table 2. Relationship between six strains of Mycoplasma hyorhinis as shown by the disc growth-inhibition technique

\begin{tabular}{lllllll} 
Strain & \multicolumn{2}{c}{ Zones of inhibition (mm.) } & * with rabbit antisera to \\
F(I)† & s7 & 200 & SK & G & GDL \\
F & 3.7 & 0 & 3.7 & 2.7 & 2.7 & 2.5 \\
S7 & 0 & 0 & 0 & 0 & 0 & 0 \\
200 & 3.5 & 0 & 4.5 & 3.5 & 3.2 & 3.5 \\
SK & 0 & 0 & 3 & 4.5 & 0 & 3 \\
G & 0 & 0 & 0 & 0 & 0 & 0 \\
GDL & 0 & 0 & 2.5 & 3 & 0 & 3.5
\end{tabular}

* Zones < I mm. are indicated with $0 . \quad \dagger_{\mathrm{F}}(\mathrm{I})$ : antiserum to strain $\mathrm{F}$ from rabbit No. $\mathrm{I}$.

Table 3. A survey of strains of Mycoplasma hyorhinis sensitive and insensitive to antiserum in disc growth-inhibition tests

\begin{tabular}{|c|c|c|c|c|c|c|c|c|c|}
\hline \multirow[b]{2}{*}{ Strain } & \multicolumn{9}{|c|}{ Presence $(+)^{*}$ or absence $(-)$ of zones of inhibition with rabbit antisera to } \\
\hline & $\mathbf{k}$ & $\mathbf{F}(\mathrm{I}) \dagger$ & $F(2) \dagger$ & 200 & SK & GDL & G & w & s7 \\
\hline $\mathbf{K}$ & + & + & + & + & + & + & + & + & + \\
\hline $\mathbf{F}$ & + & + & + & + & + & + & + & + & - \\
\hline 200 & + & + & + & + & + & + & + & - & - \\
\hline SK & - & - & + & + & + & + & - & - & - \\
\hline GDL & - & - & - & + & + & + & - & - & - \\
\hline G & - & - & - & - & - & - & - & - & - \\
\hline$w$ & - & - & - & - & - & - & - & - & - \\
\hline $\mathrm{s}_{7}$ & - & - & - & - & - & - & - & - & - \\
\hline F/D & - & - & - & - & - & - & - & - & - \\
\hline
\end{tabular}

Variations in the results of disc growth-inhibition tests. A liquid culture of the antiserum-sensitive strain $\mathbf{F}$ was mixed with various dilutions of a culture containing organisms (F/D or SK) insensitive to the antiserum used. The mixtures were then inoculated on agar medium and tested by disc growth-inhibition. The results are shown in Table 4. Variation in the proportion of antiserum-sensitive to insensitive organisms resulted in either the production of a zone of growth inhibition or the absence of such a zone. A similar variation in antiserum sensitivity was observed to occur on continued passage of a strain. Thus, the F/D strain emerged as an antiserum-resistant derivative after more than $\mathrm{I} 2$ subcultures of the sensitive $\mathrm{F}$ strain in liquid medium. Either this or variation in the proportion of antiserum-sensitive to resistant organisms in a liquid culture might explain why the results of disc growth-inhibition tests with 
some of the strains were different from those observed previously (Dinter et al. 1965). Since that report, these strains have been subcultured several times in liquid media.

Since the growth of some mycoplasma strains was not inhibited by antiserum in discs on solid medium, but was inhibited by the same antiserum in liquid medium, it was thought that decrease in the concentration of agar might result in the formation of a zone of growth inhibition. However, with strain s7 no zone was formed on solid medium containing as little as $0.1 \%$ agar. Further, the size of zones produced by antiserum in tests with strain $F$ was not significantly increased by using this low concentration of agar. The inhibition of mycoplasma growth by antiserum in a disc was not changed by the substitution of agarose for agar, the addition of I \% glucose to the agar, the use of unheated antiserum, or the addition of fresh guinea-pig serum to the antiserum.

Table 4. Zone development with mixtures of Mycoplasma hyorhinis organisms sensitive and insensitive to antiserum

No. of c.f.u.* of indicated organism in mixture of

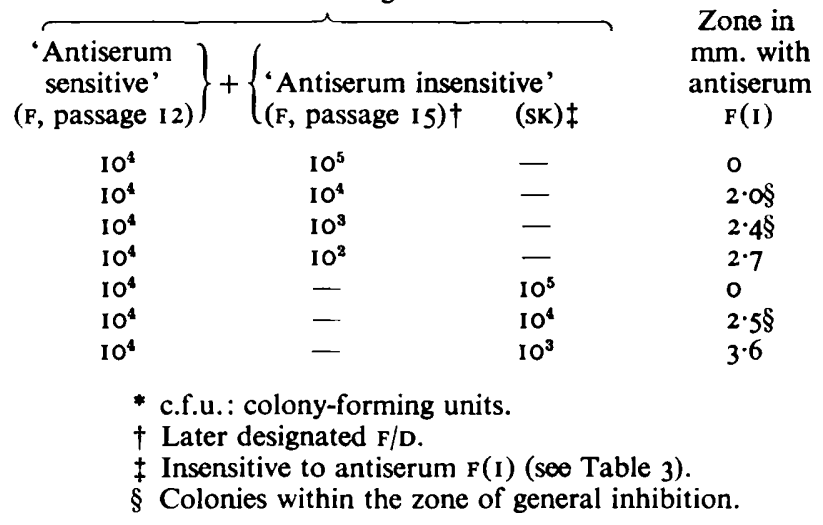

Formation of precipitation rings. Disc growth-inhibition tests were incubated up to Io days after inoculation. Within this time, in some of the tests, rings of precipitate occurred in the agar medium around the discs containing antisera (Pl. I, fig. I). The antisera had been absorbed with concentrated medium before use, and when they were tested by gel diffusion they did not produce precipitation lines with concentrated extracts of the agar medium. Thus, the rings were produced apparently by the interaction of antiserum with antigens released into the agar from organisms in the colonies. The total number of rings produced by each mycoplasma strain exposed to various antisera, and by each antiserum exposed to various strains, was counted. The largest number of rings ( 8 to II) was formed by antisera to strains 200 , SK and GDL, and the smallest number ( $\mathrm{I}$ to 3 ) by antisera to the $\mathrm{w}$ and $\mathrm{s} 7$ strains. Within the group of 'antiserum sensitive' strains, the largest number of rings (up to 13 ) was produced by the $\mathrm{K}$ strain, and the smallest number (4) by the GDL strain. A small number of rings (I to 4), often poorly developed, was produced by 'antiserum insensitive' strains. Thus, a rough correlation was apparent between the number of precipitation rings, the strength of antisera and susceptibility of the strains to antibodies.

Sensitivity of strains to polymyxins and other antibiotics. The occurrence of a greater 
number of precipitation rings with antiserum-sensitive mycoplasma strains suggested that the limiting membrane of these strains was more susceptible to damage than was the membrane of antiserum-insensitive strains. To study this further, all the mycoplasma strains were tested against various antibiotics, some of which were known to inhibit the growth of bacteria by damaging their membranes. In preliminary tests commercial paper discs were used which contained the various antibiotics listed in Methods. Apart from the exceptions noted below, the growth of all the strains was inhibited by the antibiotics in the doses tested. Bacitracin and streptomycin did not inhibit the growth of any of the strains.

In preliminary tests with commercial paper discs the zones of growth inhibition produced by colistin sodium methanesulphonate (colistin: a polymyxin type) were greater in diameter when the agar medium was at $\mathrm{pH} 7.8$ than when it was at $\mathrm{pH} 7.2$, and in all subsequent tests the media were adjusted to $\mathrm{pH} 7 \cdot 8$. The results of the preliminary tests showed also that the antiserum-sensitive strains were also sensitive to polymyxins, while the antiserum-insensitive strains were resistant (Pl. I, fig. 2).

Quantitative tests were then made in which various concentrations of colistin and

Table 5. Inhibition of growth of strains of Mycoplasma hyorhinis by various amounts of colistin sodium methanesulphonate in discs

\begin{tabular}{|c|c|c|c|c|c|c|c|}
\hline \multirow[b]{2}{*}{ Strain } & \multicolumn{6}{|c|}{ Zones of inhibition (mm.) with colistin ( $\mu \mathrm{g} . / \mathrm{disc})$} & \multirow{2}{*}{$\begin{array}{c}\text { Inhibition } \\
\text { (mean) }\end{array}$} \\
\hline & 0 & 36 & 72 & I 44 & 288 & Standard * & \\
\hline $\mathbf{K}$ & 0 & 3 & 4 & 6.5 & 10 & 3 & $5 \cdot 3$ \\
\hline $\mathbf{F}$ & o & $2 \cdot 5$ & 3 & 6 & 8 & 3 & $4 \cdot 5$ \\
\hline 200 & 0 & 3 & 5 & 7 & n.t. & 5 & 5 \\
\hline SK & 0 & 3.5 & 5 & 9 & 15 & 5 & $7 \cdot 5$ \\
\hline GDL & 0 & $2 \cdot 5$ & 4.5 & 6 & 10 & 4 & $5 \cdot 4$ \\
\hline F/D & 0 & $1 \cdot 5$ & I & 3 & 6.5 & 0 & $2 \cdot 4$ \\
\hline s7 & 0 & 0 & I. 5 & $4 \dagger$ & 4 & $1 \cdot 5$ & $2 \cdot 2$ \\
\hline G & 0 & 0 & 0 & $2 \dagger$ & 3.5 & 0 & $I \cdot I$ \\
\hline$w$ & 0 & 0 & $I \cdot 5$ & $3 \dagger$ & 6.5 & 0 & $2 \cdot 2$ \\
\hline
\end{tabular}

Table 6. Inhibition of growth of strains of Mycoplasma hyorhinis by various amounts of polymyxin $B$ in discs

\begin{tabular}{|c|c|c|c|c|c|c|c|}
\hline \multirow[b]{2}{*}{ Strain } & \multicolumn{6}{|c|}{ Zones of inhibition (mm.) with polymyxin ( $\mu \mathrm{g} . / \mathrm{disc})$} & \multirow{2}{*}{$\begin{array}{c}\text { Inhibition } \\
\text { (mean) }\end{array}$} \\
\hline & o & 25 & 50 & 100 & 200 & Standard* & \\
\hline $\mathbf{K}$ & o & $2 \cdot 5$ & 5 & 8 & 9 & 4 & $5 \cdot 7$ \\
\hline $\mathbf{F}$ & 0 & $2 \cdot 5 \dagger$ & 3 & 5 & 7 & 4 & $4 \cdot 3$ \\
\hline 200 & 0 & $2 \dagger$ & $3 \cdot 5$ & 6 & $7 \cdot 5$ & 3 & 4.4 \\
\hline SK & 0 & 2.5 & 5 & 7 & I I & 3 & $5 \cdot 7$ \\
\hline GDL & 0 & $2 \cdot 5$ & $3 \cdot 5$ & $6 \cdot 5$ & 9 & $2 \cdot 5$ & 4.8 \\
\hline $\mathbf{F} / \mathbf{D}$ & 0 & 0 & 1.5 & 2 & 4.5 & 0 & $1 \cdot 6$ \\
\hline s7 & 0 & 0 & 1.5 & 2.5 & 4 & 1.5 & 1.9 \\
\hline G & 0 & 0 & 0 & 0 & 0 & $2.5 \dagger$ & 0.5 \\
\hline$w$ & 0 & 0 & 0 & 0 & $I \cdot 5$ & 0 & 0.3 \\
\hline
\end{tabular}


polymyxin $B$ were tested against all the nine mycoplasma strains. The results are shown in Tables 5 and 6 . The mean size of zones of inhibition produced by different concentrations of colistin was a little greater than the mean size of zones produced by polymyxin B. Again, the size of zones with antiserum-sensitive strains was greater than with antiserum-insensitive strains. However, within the group of antiserum-sensitive or polymyxin-sensitive strains there was no close correlation between the sensitivity of a mycoplasma strain to antisera and the degree of its inhibition by polymyxins (compare Tables 5 and 6 with Table 3). The critical concentration of polymyxin which differentiated sensitive from insensitive strains was $72 \mu \mathrm{g}$. colistin/disc and $25 \mu \mathrm{g}$. polymyxin $\mathrm{B} /$ disc.

In m.i. tests $100 \mu \mathrm{g}$. colistin $/ \mathrm{ml}$. decreased the number of colour-changing units (c.c.u. $/ \mathrm{ml}$.) of all the mycoplasma strains by the same amount, and $250 \mu \mathrm{g}$. colistin $/ \mathrm{ml}$. completely or almost completely inhibited the metabolism of all the strains. Thus, the inhibition tests in liquid medium did not reveal differences between polymyxinsensitive and polymyxin-insensitive strains.

Table 7. Concentration of horse serum in agar medium required for the growth of strains of Mycoplasma hyorhinis

\begin{tabular}{|c|c|c|c|c|c|c|}
\hline \multirow[b]{2}{*}{ Strain } & \multicolumn{6}{|c|}{$\begin{array}{l}\text { Growth of strains* on agar medium containing indicated } \\
\text { concentrations }(\% \mathrm{v} / \mathrm{v}) \text { of horse serum }\end{array}$} \\
\hline & 0 & 2 & 4 & 5 & 10 & 20 \\
\hline $\mathbf{F}$ & . & . & . & $<2$ & 3 & $>3$ \\
\hline SK & . & . & . & $<2$ & 3 & $>3$ \\
\hline 200 & . & . & $<2$ & 2 & 3 & $>3$ \\
\hline $\mathbf{K}$ & . & . & $<2$ & 2 & $>3$ & . \\
\hline GDL & . & $<2$ & 3 & $>3$ & . & . \\
\hline$F / D$ & $<2$ & 2 & 3 & $>3$ & . & . \\
\hline s7 & $<2$ & 3 & $>3$ & . & . & . \\
\hline G & $<2$ & 3 & $>3$ & . & . & . \\
\hline w & $<2$ & 3 & $>3$ & . & . & . \\
\hline
\end{tabular}

* $<2,2$ or $3,>3=$ respectively, no colonies, few colonies, many colonies developing after inoculation of agar medium with liquid cultures diluted 100-fold (2) or 1000-fold (3).

Influence of horse serum concentration on mycoplasmal growth. During these studies it was noted that some of the mycoplasma strains were able to grow on agar medium containing less than $20 \%(\mathrm{v} / \mathrm{v})$ horse serum. To investigate this, each strain was inoculated on agar medium which contained various concentrations of horse serum. Colonies of antiserum-insensitive strains developed on agar medium containing low concentrations of horse serum, while colonies of sensitive strains developed only on agar with higher concentrations (Table 7). However, within the group of antiserumsensitive strains there was no correlation between the sensitivity to antisera and the degree of dependence upon horse serum for growth, although the GDL strain seemed to be least dependent (compare Table 7 with Table 3 ).

When the various mycoplasma strains were grown in liquid medium containing $5 \%(\mathrm{v} / \mathrm{v})$ horse serum, there was no difference in the amount of growth, in terms of colony-forming units, as between antiserum-sensitive and antiserum-insensitive strains. Furthermore, addition of various amounts of colistin to such a medium did not elicit detectable differences between the strains. 
Influence of $p H$ value of agar medium on mycoplasmal growth. Eight of the mycoplasma strains were each inoculated on agar medium at $\mathrm{pH} 7.8$ and at $\mathrm{pH} 6.5$. The number of colonies was counted after incubation for 4 days at $37^{\circ}$; the results are shown in Table 8. In general, the number of colonies of antiserum-sensitive strains which developed on agar medium at $\mathrm{pH} 6.5$ was less than the number of colonies of antiserum insensitive strains. The mycoplasma strains were inoculated also into liquid medium at the two $\mathrm{pH}$ values. Samples were removed after various times of incubation at $37^{\circ}$ and inoculated on solid medium. As judged by colony counts, all strains multiplied less in liquid medium at $\mathrm{pH} 6.5$ than at $\mathrm{pH} 7.8$. However, the growth (c.f.u./ml.) of antiserum-sensitive or antiserum-insensitive strains at $\mathrm{pH} 6.5$ was the same.

\begin{tabular}{|c|c|}
\hline Strain & $\begin{array}{c}\text { Decrease } \\
\left(\log _{10}\right) \\
\text { in number } \\
\text { of colonies* }\end{array}$ \\
\hline $\mathbf{K}$ & $>3$ \\
\hline $\mathbf{F}$ & $>3$ \\
\hline 200 & $>4$ \\
\hline sK & 4 \\
\hline GDL & $>2$ \\
\hline G & 2 \\
\hline s7 & Nil \\
\hline $\mathbf{F} / \mathbf{D}$ & Nil \\
\hline
\end{tabular}

Size of mycoplasma organisms. The possibility that antiserum-sensitive strains consisted of organisms of a different size from antiserum-insensitive strains was considered. Liquid cultures of strains $\mathrm{F}$ and $\mathrm{s} 7$ were filtered through Millipore filters of different pore sizes; the proportion of organisms of each strain which passed through each filter was the same. Thus, there was no evidence to suggest that there was a difference in the size of organisms of the different mycoplasma strains.

\section{DISCUSSION}

The differences in the sensitivity of the nine Mycoplasma hyorhinis strains to specific antisera, polymyxins or $\mathrm{pH} 6.5$ were observed only when they were grown on solid medium. It was not possible to differentiate the strains into sensitive and insensitive ones when the organisms were grown in liquid medium. Furthermore, growth of sensitive strains, as defined above, on solid medium required higher concentrations of horse serum than did the growth of insensitive strains. Such a difference was not seen when the various strains were grown in liquid medium.

The concept of sensitivity as used in the present paper is empirical. Those strains that were inhibited by all the antisera were regarded as most sensitive, those inhibited by fewer antisera as less sensitive and those not inhibited by any antisera as antiserum resistant. It is not known whether individual organisms are either sensitive or resistant and remain so on repeated passage, in which case the sensitivity of a particular mycoplasma strain would be dependent upon the proportions of the different organisms in 
the culture, or whether sensitive organisms changed to insensitive, and vice versa. It is also difficult to explain why the various phenomena were observed only in tests on solid medium. Possibly strains that appeared insensitive to antiserum or polymyxins on solid medium were sensitive in liquid medium because of the opportunity for more intimate contact with antibody or antibiotic. Whatever the reason for the phenomena occurring on solid medium only, it seems clear that the differences between sensitive and insensitive strains, whether the sensitivity is to antiserum or polymyxins, may be related to the mycoplasma membrane. There is evidence that growth-inhibitory antisera are directed against antigens in or on the mycoplasma membrane (Williams \& Taylor-Robinson, 1967). The mechanism of mycoplasmal growth-inhibition by polymyxins is unknown, but it is known that polymyxins inhibit the growth of bacteria by damaging their membranes (Newton, 1956; Sebek, 1967). Well-defined precipitation rings occurred in disc growth-inhibition tests in which Mycoplasma hyorhinis growth was inhibited by antiserum. Such precipitation is not confined to tests with $M$. hyorhinis since, occasionally, similar precipitation rings have been observed in studies of other mycoplasma species. In the case of $M$. hyorhinis it seems that the phenomenon is indicative of membrane damage with lysis of the organisms. Lysis with liberation of antigens must have occurred, since in tests with antiserumin sensitive strains, although antigen was potentially available because of profuse colony growth, rings of precipitation were few in number and were poorly developed. Thus, the theory may be put forward that specific antibodies attach to the mycoplasma membrane, damage it and cause leakage of specific antigens into the agar, and that the membranes of sensitive organisms are more susceptible to damage by antibodies in this way than those of insensitive strains.

Newton (1956) analysed cell walls from a polymyxin-sensitive and a polymyxinresistant strain of Pseudomonas aeruginosa. A difference in the phospholipid content of the two strains was thought to be a factor contributing to the selective action of polymyxin, the phospholipid content being greater in the sensitive than in the resistant strain. The dependence for growth of some of our antiserum-sensitive mycoplasma strains on high concentrations of horse serum does not appear to contradict this. Horse serum supplements the medium with protein, cholesterol and phospholipid, known to be required for the reproduction of parasitic mycoplasma strains (Razin, Cosenza \& Tourtellotte, 1967), and which are incorporated into the mycoplasma membrane. It has been shown by Sobeslavsky, Prescott, James \& Chanock (1966) that lipid in the membrane of Mycoplasma pneumoniae is concerned in growth inhibition by antiserum. These authors were able to block the effect of growthinhibitory antiserum with lipid extracted from this organism. Possibly quantitative changes in the phospholipid composition of $M$. hyorhinis organisms could account for their changes in antiserum sensitivity. A change in the antiserum sensitivity of a strain on serial passage might be due to variation in the composition of the mycoplasma membrane. The fact that the growth of antiserum-sensitive strains in the present experiments was suppressed on agar medium at $\mathrm{pH} 6.5$ may be attributable also to the phospholipid content of their membranes. It has been shown for some bacteria (van Deenan, 1965) that a change in their phospholipid distribution could be attributed to a decrease of the $\mathrm{pH}$ value of the medium. Such could be the case with mycoplasmas.

Hayflick \& Stanbridge (1967) studied a strain of Mycoplasma hyorhinis and considered that it contained a population of organisms, some sensitive and others resistant 
to antiserum. They found colonies produced by apparently resistant organisms in the zone of growth inhibition produced by antiserum-sensitive organisms. We have not observed this particular phenomenon to occur naturally although it has been possible to reproduce it experimentally with mixtures of sensitive and insensitive strains (Table 4). However, our findings are consistent with the concept of antiserum-sensitive and resistant organisms, and it seems reasonable to postulate that these phenomena are attributable to changes in the composition of the mycoplasma membranes.

We thank Miss Maud Söderberg and Mrs Susan Beveridge for excellent technical assistance.

\section{REFERENCES}

Clyde, W. A., JUN. (I964). Mycoplasma species identification based upon growth inhibition by specific antisera. J. Immun. 92, 958.

van Deenan, L. K. M. (1965). Phospholipids and biomembranes. In Progress in the Chemistry of Fats and other Lipids. Ed. by R. T. Holman. Vol. 8. Oxford: Pergamon Press.

Dinter, Z., Danielsson, D. \& BaKos, K. (1965). Differentiation of porcine mycoplasma strains. J. gen. Microbiol. 41, 77.

Hayflick, L. (1965). Tissue cultures and mycoplasmas. Tex. Rep. Biol. Med., Suppl. I to Vol. 23, p. 285.

Hayflick, L. \& Stanbridge, E. (1967). Isolation and identification of mycoplasma from human clinical materials. Ann. N.Y. Acad. Sci. 143, 608.

Newton, B. A. (1956). The properties and mode of action of the polymyxins. Bact. Rev. $20,14$.

Razin, S., Cosenza, B. J. \& Tourtellotte, M. E. (1967). Filamentous growth of mycoplasma. Ann. N.Y. Acad. Sci. 143, 66.

SebeK, O. K. (1967). Polymyxins and circulin. In Antibiotics, Ed. by D. Gottlieb and P. D. Shaw. Vol. I, p. 142. Berlin-Heidelberg-New York: Springer-Verlag.

Sobeslavsky, O., Prescott, B., James, W. D. \& Chanock, R. M. (1966). Isolation and characterization of fractions of Mycoplasma pneumoniae. II. Antigenicity and immunogenicity. J. Bact. 9r, 2126.

SwITzER, W. P. (1955). Studies on infectious atrophic rhinitis. IV. Characterization of a pleuropneumonia-like organism isolated from the nasal cavities of swine. Am. J. vet. Res. 16, 540.

TAYLOR-RoBINSON, D. \& DinTER, Z. (I968). Unexpected serotypes of mycoplasmas isolated from pigs. J. gen. Microbiol. 53, 221.

Taylor-Robinson, D., Purcell, R. H., Wong, D. C. \& Chanock, R. M. (ig66). A colour test for the measurement of antibody to cortain mycoplasma species based upon the inhibition of acid production. J. Hyg., Camb. 64, $9 \mathrm{I}$.

Williams, M. H. \& TAYLoR-Robinson, D. (1967). Antigenicity of mycoplasma membranes. Nature, Lond. 215, 973.

\section{EXPLANATION OF PLATE}

Fig. 1. Disc growth-inhibition tests with the $\mathrm{K}$ strain of Mycoplasma hyorhinis. Zones of inhibition and rings of precipitation with, from left to right, rabbit antisera SK, $F$ and 200. Day 9 after inoculation.

Fig. 2. Disc growth-inhibition tests with the sensitive $K$ strain (above) and the insensitive $F / D$ strain (below) of Mycoplasma hyorhinis. Discs on left contained rabbit antiserum 200 (undiluted); discs on right contained colistin (900 i.u.). Day 4 after inoculation. 
Journal of General Microbiolog.', Vol. 57, No. 2

Plate I

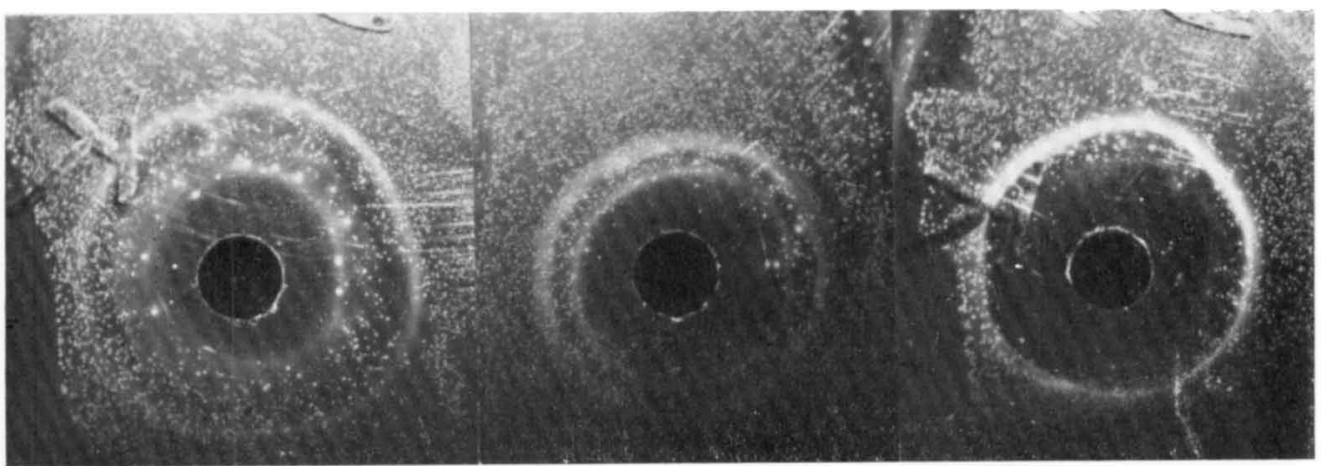

Fig I

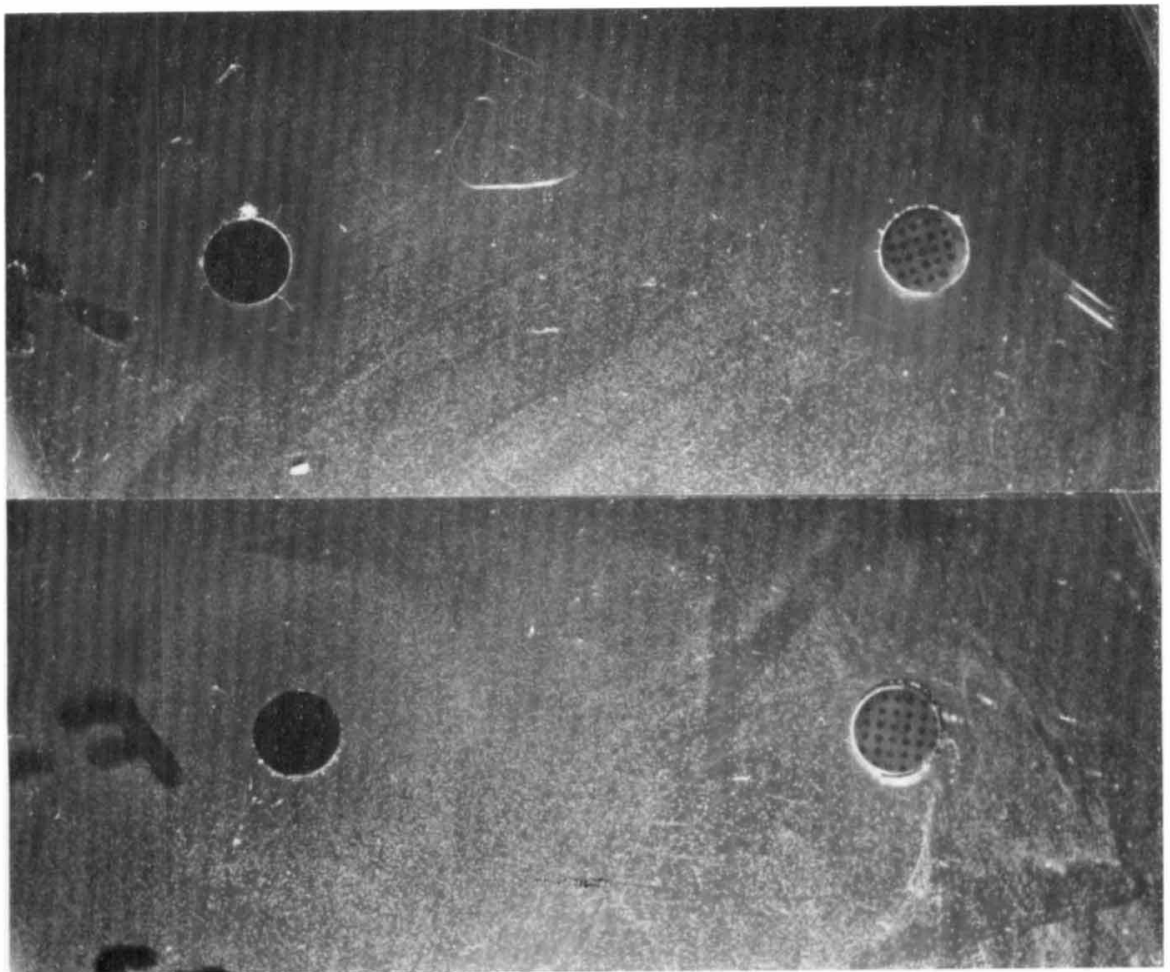

Fig. 2 